1 SLEEP DISTURBANCES ASSOCIATED WITH POSTTRAUMATIC STRESS DISORDER: PRESENTATION AND POLYSOMNOGRAPHIC FEATURES IN A POPULATION OF PATIENTS ADMITTED TO A SLEEP LABORATORY

${ }^{1}$ Joyce Y Zhang* ${ }^{*}$ lan E Smith, ${ }^{3}$ Irene Valero-Sanchez. ${ }^{1}$ University of East Anglia, UK; ${ }^{2}$ Royal Papworth Hospital NHS Foundation Trust, UK; ${ }^{3}$ University Hospitals of Leicester NHS Trust, UK

\subsection{6/bmjresp-2021-bssconf.1}

Introduction In this study we assess sleep complaints and objective sleep parameters in a cohort of patients with posttraumatic stress disorder (PTSD) admitted to a sleep laboratory.

Methods Retrospective study of patients with a diagnosis of PTSD who were referred for full polysomnography (PSG) in a sleep service as part of the investigation of a sleep disturbance. Demographic data, presenting complaints, PSG parameters, subjective levels of sleepiness, sleep diary, medication and diagnoses were recorded.

Result The sample included 30 patients, 46.7\% female 53.3\% male, mean age 45 years, (SD 15), mean BMI 28 (SD 4.7) $\mathrm{kg} / \mathrm{m} .13$ patients presented with excess daytime sleepiness or fatigue, 11 with parasomnia, 5 with poor sleep and 1 patient with nightmare disorder. 21 had a diagnosis of depression, 12 reported insomnia and 20 nightmares. $63.3 \%$ received antidepressants.

Mean total sleep time (TST) in sleep diary was 6.5 hours. Mean Epworth score was 10 (SD 6.5). PSG parameters (expressed as mean and SD) were: Sleep latency (SL) 26.3 (35.8) min, TST 390 (130) min, sleep efficiency (SE) for time in bed $77.6(16.2) \%$, SE for sleep period of time 80.5 (16.7)\%, stages 1 and 2: 48 (13.5)\%, REM 17 (15.5)\% slow wave sleep $13(9.5) \%$, apnoea hypopnea index $7.6(9.2) / \mathrm{h}$, periodic limb movement (PLM) index 19.5 (29.9)/h. Parasomnias were recorded in 7 cases. After PSG, 8 patients were diagnosed with PLM disorder, 9 with parasomnia, 6 with sleep apnoea, 3 with insomnia. 5 had no sleep disorder identified. The presence of PLM during sleep did not correlate with the usage of antidepressants.

Discussion Both sleep onset and sleep maintenance insomnia were reported. Nightmares were frequent. The presence of PLMD was higher than expected, raising the possibility that it may be a contributor to sleep disturbances in PTSD. A case controlled study would be of value.

\section{SERVICE REVIEW: A COMPARISON OF MANUAL VS AUTO ANALYSIS SCORING USING THE NOX T3 LIMITED POLYSOMNOGRAPHIC DEVICE}

Corey Davies*, Lois Attewell, Hannah Hunt, Joelle Smith, Esme Burge, Alex Tuck. Cardiff and Vale UHB, Cardiff, UK

\subsection{6/bmjresp-2021-bssconf.2}

Introduction As physiologists we spend a vast amount of time manually analysing sleep studies. As technology advances, we are often told that the auto analysis programmes are improving with every update. We decided to find out how accurate it has become and whether it can be relied on for an accurate clinical outcome.

Methods We utilised clinical downtime during the COVID-19 pandemic to carry out a service audit between September 2019 and March 2020. 160 studies were reviewed with a comparison between automatic and manually scored AHI Pearsons co-efficient of variation and Bland-Altman analysis was carried out both to investigate the correlation of the assays as well as allowing clinical judgement into the significance.

Results We found that 90 results changed scoring classification and 70 stayed the same. A Pearsons co-efficient of variation was calculated at 0.86 indicating a very high correlation (Schober, Boer and Schwarte, 2018), however overall, we found an average AHI difference of 8.4 in the scores between auto and manual. Looking closely at the distribution on the plot it suggests that the auto analysis programme correlates highly when the AHI is $<7$ with a tightly packed area of datum however as the AHI increases so does the scatter.

Discussion We found although there is a positive correlation auto analysis is unable to operate in a small enough window to have no impact on the treatment pathway. Relying on an automatic programme would indeed 'speed up' the service but at the cost of accurate clinical science as well as physical cost on misdiagnosis.

We are aware this study is limited to the NOX T3 device and a relatively small data set so may not extend beyond these limitations.

\section{REFERENCE}

1. Schober P, Boer C, and Schwarte L. Correlation Coefficients. Anesthesia \& Analgesia 2018;126(5):1763-1768.

\section{LONG TERM IMPACT OF POOR SLEEP ON FUTURE METABOLIC AND MENTAL HEALTH: A UK BIOBANK STUDY OF 84,404 PARTICIPANTS}

${ }^{1}$ Gewei Zhu*, ${ }^{2}$ David Gunn, ${ }^{3}$ Michael Catt, ${ }^{1,3}$ Mark Birch-Machin, ${ }^{4}$ Kirstie Anderson. ${ }^{1}$ Faculty of Medical Sciences, Newcastle University, Newcastle Upon Tyne, UK: ${ }^{2}$ Unilever RandD, Colworth Science Park, Sharnbrook, UK; ${ }^{3}$ National Innovation Centre for Ageing, Newcastle upon Tyne, UK: ${ }^{4}$ Department of Neurology, Royal Victoria Infirmary, Newcastle upon Tyne, UK

\subsection{6/bmjresp-2021-bssconf.3}

Introduction Both short and long sleep duration and sleep fragmentation have immediate and long-term adverse impacts on physical and mental health. However, most population studies are based on self-reported sleep and health status rather than objective assessments. There are few longitudinal datasets that measure the impact of poor sleep over time. This study investigated the impact of objectively measured poor sleep on the long term metabolic and mental health status amongst the UK Biobank population.

Methods Sociodemographic, accelerometry and primary care records data were obtained from the UK Biobank $(n=84,404)$. Sleep duration and fragmentation was objectively assessed with accelerometry (mean age $=62.4$ years) and divided into five sleep groups: $<5$ hours, 5-6 hours, 6-7 hours, 7-8 hours and $>8$ hours. Sleep fragmentation related measurements including wake after sleep onset, activity level during the least active five hours and episodes of movement during sleep were also analysed. Binary regression models were adjusted for age, gender and Townsend deprivation score. There was then detailed assessment of the primary care records after a 6-year interval. Results A 'U-shaped' relationship was found between sleep duration and incidence of many metabolic diseases, as well as mental illnesses such as depression. Fragmented sleep and both short and long sleep duration were associated with 
increased odds ratio $(\mathrm{OR})$ of diseases including diabetes $(\mathrm{OR}=$ $1.42, \mathrm{p}<0.001)$, hypertensive disease $(\mathrm{OR}=1.22, \mathrm{p}<0.001)$, ischaemic heart disease $(\mathrm{OR}=1.12, \mathrm{p}<0.001)$ and organic mental disorder $(\mathrm{OR}=1.77, \mathrm{p}<0.001)$.

Discussion Using the largest accelerometry cohort to date, 6-8 hours of sleep along with non-fragmented sleep was associated with better, future metabolic and mental health. However, sleep duration and quality alone were not sufficiently sensitive or specific to be a stand-alone biomarker for future health status. Future studies should consider additional lifestyle and health factors.

\section{COMPARING THE SUBJECTIVE SLEEPINESS AND SOCIAL HEALTH OF HIGHER EDUCATION STUDENTS ALONGSIDE A NON-STUDENT SAMPLE}

Katrina Burrows*, Abbie Millett. University of Suffolk

\subsection{6/bmjresp-2021-bssconf.4}

Introduction This paper will evaluate the difference between Sleepiness, Loneliness and Social Connectedness between a higher education and mundane sample. Although current literature evaluates the role of Sleepiness within Higher education (Araújo et al, 2021), to the best of our knowledge there are no papers comparing a student population to a non-student sample.

A significant difference between the subjective sleepiness scores of a student and non-student population is expected, which would demonstrate that a student sample perceive themselves to be sleepier than their non-student counterparts.

Methods An online survey was administered to 202 participants via Qualtrics. 94 were non-students and 108 were higher education students. Sleepiness was measured using the Stanford Sleepiness Scale (Shahid et al, 2011), The UCLA Loneliness Scale was used to measure loneliness (Russell, 1980).

Results An independent t-test showed that there was a significant difference between students and non-students subjective sleepiness scores. $(\mathrm{t}(200)=2.23, \mathrm{p}=0.02)$ with students being significantly more like to rate themselves higher on the Stanford Sleepiness Scale. (Figure 1).

Further analysis shows that Loneliness and Subjective Sleepiness are significantly correlated for a student sample ( $\mathrm{r}$ (108) $=0.308, \mathrm{P}=0.001$ ).

Discussion This study suggests that students enrolled within higher education are more highly associated with the risks of

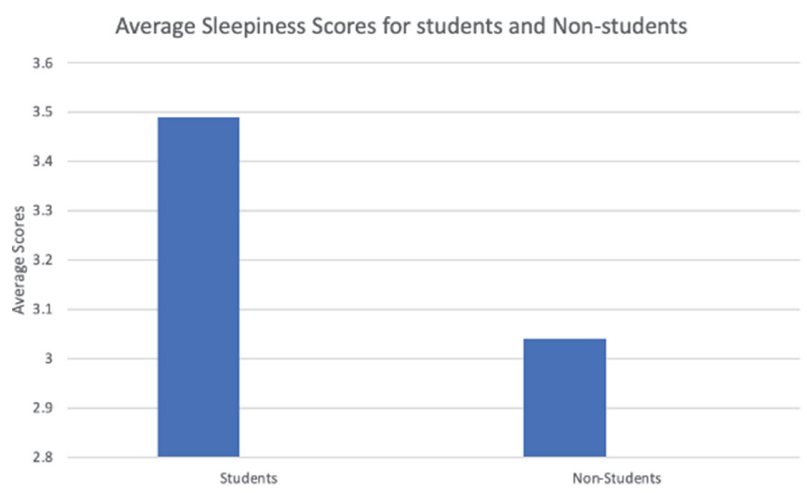

Abstract 4 Figure 1 Demonstrates the average Sleepiness scores for students and non-students
Excessive daytime Sleepiness and the resultant detrimental effects on social health. Further research needs to be performed to determine the negative effects of Sleepiness on Student's social health.

\section{INVESTIGATING METHODS OF SHARING INFORMATION TO SUPPORT ADOLESCENT SLEEP ISSUES DURING COVID-19}

Vicki Dawson*, Lisa Artis. The Sleep Charity, Doncaster, UK

\subsection{6/bmjresp-2021-bssconf.5}

Introduction Many studies outline adolescents are particularly at risk of developing sleep issues, experiencing delayed sleep onset. The aim of our study was to identify effective ways to support young people to improve sleep patterns using behavioural intervention during Covid-19 by promoting youth participation and sharing information at scale.

Method The study entailed recruiting young people with lived experience of sleep issues to create three distinct advisory boards. Through consultation it was established that Covid-19 was having a significant impact on sleep patterns in this cohort and there was an urgent need to create accessible, evidence-based sleep intervention. We established a website dedicated to sharing sleep education. A downloadable e-Book, live web chat, videos, a newsletter and resources for schools and parents were all created with the input of the boards.

Results The website launched on 1st October 2020 with the aim to reach 400 young people by the end of the project on 6 th June 2021. The project had the following reach:

Website visitors - 19,766

eBook downloads - 33,468

Newsletter signups - 1,601

Video views sharing sleep tips - 14,491

Young people receiving direct support through live chat -34

Schools receiving resource pack $-3,500$

Feedback from 50 individuals was evaluated to inform future work around the eBook and it was established that there is a strong preference for printed material, these findings were also supported by our youth advisory board.

Discussion Young people consistently told us that they want to be able to access evidence-based sleep information to support their own sleep patterns, our work lays important foundations on which to build nationally to support adolescent sleep and wellbeing at scale.

\section{EVALUATION OF A ONE-TO-ONE SLEEP SERVICE DELIVERED VIA ONLINE CLINICS BY COMMUNITY SLEEP PRACTITIONERS IN THE UK}

${ }^{1}$ Victoria Dawson*, ${ }^{2}$ Heather Elphick, 'Lisa Artis, ${ }^{2}$ Lowri Thomas, ${ }^{1}$ Claire Earley, ${ }^{1}$ Karen Tyas. ${ }^{1}$ The Sleep Charity, Doncaster, UK; ${ }^{2}$ Sheffield Children's Hospital, Sheffield, UK

\subsection{6/bmjresp-2021-bssconf.6}

Introduction Poor sleep is associated with many adverse outcomes in childhood. The most common sleep difficulty experienced by children is behavioural insomnia which responds to behavioural therapy. The Coronavirus disease 2019 (COVID19) global pandemic in 2020 has had a profound effect on children's sleep patterns. This project aimed to evaluate a one- 